\title{
ON THE ASYMPTOTIC BEHAVIOR OF SOLUTIONS NEAR AN IRREGULAR SINGULARITY
}

\author{
STEVEN BANK
}

1. Introduction. In [2], the asymptotic behavior of solutions of $n$ th-order homogeneous linear ordinary differential equations near a singular point at $\infty$, was investigated. The class of equations treated in that paper (as well as here) roughly consists of those linear equations whose coefficients are complex functions defined and analytic in unbounded sectorial regions (see $\S 2$ (a) below), and have asymptotic expansions as $x \rightarrow \infty$ in terms of real (but not necessarily integral) powers of $x$ and/or functions which are of smaller rate of growth $(\prec)$ than all powers of $x$ as $x \rightarrow \infty$. (We are using here the concept of asymptotic equivalence $(\sim)$ as $x \rightarrow \infty$, and the order relation " $\prec$," in troduced in $[5, \S 13]$.) However, it should be noted (see $[5, \S 128(\mathrm{~g})]$ ) that the class of equations treated here includes, as a special case, equations where no requirement is imposed except that each coefficient be analytic and have an asymptotic expansion (in the customary sense) of the form $\sum c_{j} x^{-\lambda_{j}}$ with $\lambda_{j}$ real and $\lambda_{j} \rightarrow+\infty$ as $j \rightarrow \infty$. (For a summary of the necessary definitions from [5], and the needed results from [2], see $\S \S 2,3$ below.) In [2], solutions were sought which were $\sim$ to complex logarithmic monomials (i.e. functions of the form, $K x^{\alpha_{0}}(\log x)^{\alpha_{1}}(\log \log x)^{\alpha_{2}} \cdots\left(\log _{q} x\right)^{\alpha_{q}}$ for complex $\alpha_{j}$ and $K$ with $K \neq 0$ ). Associated with a linear equation in the class described above, is an algebraic equation of degree at most $n$ (see $[1, \S 17]$ or $[2, \S 3(\mathrm{e})])$, which is a generalization of the classical "indicial equation at $\infty$." In $[2, \S \S 5,11]$, it was shown that if $p$ is the degree of this corresponding algebraic equation, then there are precisely $p$ complex logarithmic monomials of the form $M_{j}=x^{\beta_{j}}(\log x)^{\gamma_{j}}(j=1, \cdots, p)$, where $M_{i}$ is not $\sim M_{j}$ if $i \neq j$, such that any solution of the differential equation which is $\sim$ to a complex logarithmic monomial is $\sim$ to a constant multiple of some $M_{j}$, and there exist solutions $g_{i} \sim M_{i}$ such that $\left\{g_{1}, \cdots, g_{p}\right\}$ is a linearly independent set. When $p=n$ (which is a generalization of the notion of regular singularity at $\infty$ ), this result is an asymptotic analog of one part of the classical Fuchs Regularity Theorem. (See $[4$, p. 365] for a complete discussion of Fuchs' Theorem.)

When $p<n$ (which generalizes the situation of an irregular singularity at $\infty)$, a natural question is raised; namely, what is the

\footnotetext{
Received by the editors April 141966.
} 
asymptotic behavior of the remaining $n-p$ solutions in a fundamental set? In this paper we answer this question in the case $p=n-1$, by proving (see $\S 4$ below) that when $p=n-1$, there is a solution $g^{*}$, such that $\left\{g_{1}, \cdots, g_{n-1}, g^{*}\right\}$ is a fundamental set, and such that $g^{*}$ is either of smaller rate of growth as $x \rightarrow \infty$ than all powers of $x$, or is of larger rate of growth as $x \rightarrow \infty$ than all powers of $x$. This result is proved using the classical technique of reduction of order as well as results in [3] and [6] concerning the asymptotic behavior of solutions of certain first-order linear equations.

2. Concepts from [5]. (a) $[5, \S 94]$ : Let $a$ and $b$ be real numbers, $-\pi \leqq a<b \leqq \pi$. For each nonnegative real-valued function $g$, defined on $(0,(b-a) / 2)$, let $V(g)$ be the union (over $\delta \in(0,(b-a) / 2)$ ) of all sectors, $a+\delta<\arg (x-h(\delta))<b-\delta$, where $h(\delta)=g(\delta) \exp (i(a+b) / 2)$. The set of all $V(g)$ (for all choices of $g$ ) is denoted $F(a, b)$, and is a filter base which converges to $\infty$. A statement is said to hold except in finitely many directions in $F(a, b)$ (briefly, e.f.d. in $F(a, b)$ ) if there are finitely many points $r_{1}<r_{2}<\cdots<r_{q}$ in $(a, b)$, such that the statement holds in each of $F\left(a, r_{1}\right), F\left(r_{1}, r_{2}\right), \cdots, F\left(r_{q}, b\right)$ separately.

(b) $[5, \S 13]:$ If $f$ is analytic in some $V(g)$, then $f \rightarrow 0$ in $F(a, b)$ means that for any $\epsilon>0$ there is a $g_{1}$ such that $|f(x)|<\epsilon$ for all $x \in V\left(g_{1}\right)$. The statement $f \prec 1$ in $F(a, b)$ means that in addition to $f \rightarrow 0$, all functions $\theta_{j}^{k} f \rightarrow 0$ where $\theta_{j} f=x \log x \cdots \log _{j-1} x f^{\prime}$. The statements $f_{1} \prec f_{2}, f_{1} \sim f_{2}$ and $f_{1} \approx f_{2}$ mean respectively, $f_{1} / f_{2} \prec 1, f_{1}-f_{2} \prec f_{2}$ and $f_{1} \sim c f_{2}$ for some constant $c \neq 0$. An important property of the order relation $\prec$, (proved in $[5, \S 28]$ ) is that $f \prec 1$ implies $\theta_{j} f \prec 1$ for all $j$, so in particular, $x f^{\prime} \prec 1$.

(c) $[5, \S 49]$ : A logarithmic domain of rank zero, (briefly an $\mathrm{LD}_{0}$ ) over $F(a, b)$, is a complex vector space $E$ of functions (each analy tic in some $V(g))$, which contains the constants, and such that any finite linear combination of elements of $E$, with coefficients which for some $q \geqq 0$ are functions of the form $c x^{\alpha_{0}}(\log x)^{\alpha_{1}} \ldots\left(\log _{q} x\right)^{\alpha_{q}}$ (for real $\alpha_{j}$ ), is either $\sim$ to a function of this latter form or is trivial (i.e. $\prec x^{\alpha}$ for all real $\alpha$ ).

3. A result from [2]. Let $\Omega(y)$ be an $n$ th-order linear differential polynomial with coefficients in an $\mathrm{LD}_{0}$ over $F(a, b)$. If $\theta$ is the operator $\theta y=x y^{\prime}, \Omega(y)$ may be written $\Omega(y)=\sum_{j=0}^{n} B_{j}(x) \theta^{j} y$, where the functions $B_{j}$ belong to an $\mathrm{LD}_{0}$. We assume $B_{n}$ is nontrivial. By dividing through by the highest power of $x$ which is $\sim$ to a coefficient, $B_{j}$, we may assume there is an integer $p \geqq 0$ such that $B_{p} \approx 1, B_{j} \prec 1$ or $\approx 1$ for each $j$, while for $j>p, B_{j} \prec x^{-\delta}$ for some $\delta>0$. It is proved 
in $[2, \S 11]$ (using results obtained in [1] and [7]) that there exist $p$ complex logarithmic monomials $M_{1}, \cdots, M_{p}$, where each is of the form $x^{\alpha}(\log x)^{\beta}$, and $M_{i} \approx M_{j}$ if $i \neq j$, such that any solution of $\Omega(y)=0$ which is $\sim$ to a complex logarithmic monomial is $\approx M_{i}$ for some $i$. and such that e.f.d. in $F(a, b)$, the equation $\Omega(y)=0$ possesses solutions $g_{1}, \cdots, g_{p}$ with $g_{i} \sim M_{i}$. If $I$ is an interval such that $g_{1}, \cdots, g_{p}$ exist in $F(I)$, we say $\left\{g_{1}, \cdots, g_{p}\right\}$ is a complete logarithmic set of solutions in $F(I)$. The functions $g_{1}, \cdots, g_{p}$ have the property that if for some constants $c_{1}, \cdots, c_{p}$ the function $\sum_{i=1}^{p} c_{i} g_{i}$ is trivial then all the $c_{i}$ are 0 .

4. The main theorem here. We will prove the following

ThEOREM. Let $\Omega(y)=\sum_{i=0}^{n} B_{i}(x) \theta^{i} y$ be a linear differential polynomial where the functions $B_{i}$ belong to an $\mathrm{LD}_{0}$ over $F(a, b)$, and where $B_{n-1} \approx 1, B_{j} \prec 1$ or $\approx 1$ for all $j, B_{n} \prec x^{-\delta}$ for some $\delta>0$ and $B_{n}$ is nontrivial. Then $\left(-B_{n-1} /\left(x B_{n}\right)\right) \sim c x^{-1+t}$ for some constant $c \neq 0$ and $t>0$. (This follows from the asymptotic properties of $B_{j}$, and the definition of $\left.\mathrm{LD}_{0}.\right)$ Let $f(\phi)=\cos (t \phi+\arg c)$ for $-\pi<\phi<\pi$. Then if $\left(a_{1}, b_{1}\right)$ is any subinterval of $(a, b)$ on which $f(\phi)<0$ (respectively $f(\phi)>0$ ), then e.f.d. in $F\left(a_{1}, b_{1}\right)$ the equation $\Omega(y)=0$ has a fundamental set of solutions $\left\{g_{1}, \cdots, g_{n-1}, g^{*}\right\}$, where $\left\{g_{1}, \cdots, g_{n-1}\right\}$ is a complete logarithmic set and $g^{*} \prec x^{\alpha}$ for all $\alpha$ (respectively $g^{*} \succ x^{\alpha}$ for all $\left.\alpha\right)$.

The proof of this theorem will be based on a sequence of lemmas, and will be concluded in $\$ 12$.

5. Notation. If $H$ is a nonzero solution of an $n$ th-order homogeneous linear differential equation $\Lambda(y)=0$, then under the change of dependent variable $y=H u, z=\theta u$ followed by division by $H$, we obtain an $(n-1)$ st-order equation denoted $(H ; \Lambda)(z)=0$. By induction we denote $\left(H_{i} ;\left(H_{i-1}, \cdots, H_{1} ; \Lambda\right)\right)$, if defined, by $\left(H_{i}, H_{i-1}, \cdots\right.$, $\left.H_{1} ; \Lambda\right)$, where if $i=0$, the latter is taken to be $\Lambda$ itself.

6. Uniform hypothesis. Let $\Omega$ satisfy the hypothesis of $\$ 4$. Let $I$ be any interval such that a complete logarithmic set $\left\{g_{1}, \cdots, g_{n-1}\right\}$ exists in $F(I)$ (see $\S 3$ ). Define functions $h_{1}, \cdots, h_{n-1}$ as follows: $h_{1}=g_{1}$ and $h_{i+1}=\left(\theta \circ h_{i}^{\#} \circ \cdots \circ \theta \circ h_{1}^{\mu}\right)\left(g_{i+1}\right)$, where $h^{*}$ is the operator $h^{\#} y=y / h$. Let $\Lambda_{i}$ be the operator $\Lambda_{i}(y)=\left(\theta \circ h_{i}^{\#} \circ \cdots \circ \theta \circ h_{1}^{\#}\right)(y)$.

It is proved in $[2, \S 12(\mathrm{~B})]$ that

(A) $h_{i}$ is $\sim$ to a complex logarithmic monomial (whence by simple computation, $\theta^{i} h_{i} / h_{i} \prec 1$ or $\approx 1$ for all $i$ and $j$ ).

(B) $\left(h_{i}, h_{i-1}, \cdots, h_{1} ; \Omega\right)$ is defined for all $i=0, \cdots, n-1$. 
7. Lemma. Assume the hypothesis of $\$ 6$. For each $i$, let

$$
\left(h_{i}, \cdots, h_{1} ; \Omega\right)(y)=\sum_{z=0}^{n-i} B_{i, 3} \theta^{s} y .
$$

Then, for each $i$,

(a) $B_{i, n-i}=B_{n}$, and

(b) $B_{i, n-i-1} \sim B_{n-1}$ in $F(I)$.

Proof. By simple computation,

$$
B_{i+1, s}=\sum_{j=s+1}^{n-i} B_{i j}\left(\begin{array}{c}
j \\
s+1
\end{array}\right)\left(h_{i+1}\right)^{-1} \theta^{j-(s+1)} h_{i+1} .
$$

For $i=0$, (a) and (b) are clear. We proceed by induction, and assume (a) and (b) for $i$. By (1), $B_{i+1, n-(i+1)}=B_{i, n-i}$ which is $B_{n}$ by induction hypothesis, proving (a) for $i+1$. (b) follows for $i+1$, from (1) and the asymptotic relations, $B_{i, n-i-1} \sim B_{n-1} \approx 1, B_{i, n-i}=B_{n} \prec x^{-\delta}$ and $\left(h_{i+1}\right)^{-1} \theta h_{i+1} \prec 1$ or $\approx 1$ (by $\S 6(\mathrm{~A})$ ).

8. Lemma. Assume the hypothesis of $\$ 6$. Then:

(a) There exists an analytic function $W \sim\left(-B_{n-1} / x B_{n}\right)$ such that the functions $z_{0}=\exp \int W$ (where $\int W$ stands for any primitive of $W$ in $F(I))$, are solutions of $\left(h_{n-1}, \cdots, h_{1} ; \Omega\right)(z)=0$.

(b) $W \sim c x^{-1+t}$ for some constants $t>0$ and $c \neq 0$.

(c) The function $f(\phi)=\cos (t \phi+\arg c)$ has only finitely many zeros in $(-\pi, \pi)$.

Proof. (a) By Lemma 7 , (for $i=n-1)$, the equation $\left(h_{n-1}, \cdots, h_{1}\right.$; $\Omega)(z)=0$ is $B_{n} \theta z+E z=0$ (where $E \sim B_{n-1}$ ), and hence is equivalent to the equation $z-\left(z^{\prime} / W\right)=0$ where $W=-E /\left(x B_{n}\right)$. Thus (a) follows immediately.

(b) and (c) are obvious.

9. Lemma. Assume the hypothesis of $\$ 6$ and let $W$ be as in Lemma 8 . Then if $g$ is any function such that $\Lambda_{n-1}(g)=\exp \int W$, we have $\Omega(g)=0$.

Proof. From the definition of $(H ; \Lambda)$ it is clear that,

(A) If $z_{0}=\left(\theta \circ H^{H}\right)\left(y_{0}\right)$ is a solution of $(H ; \Lambda)(z)=0$, then $y=y_{0}$ is a solution of $\Lambda(y)=0$.

Let $g$ be a function such that $\Lambda_{n-1}(g)=\exp \int W$. Thus $\exp \int W$ $=\left(\theta \circ h_{n-1}^{\#}\right)\left(\Lambda_{n-2}(g)\right)$, and by Lemma $8, z_{0}=\exp \int W$ is a solution of $\left(h_{n-1}, \cdots, h_{1} ; \Omega\right)(z)=0$. Hence by $(\mathrm{A}), y_{1}=\Lambda_{n-2}(g)$ is a solution of $\left(h_{n-2}, \cdots, h_{1} ; \Omega\right)(y)=0$. But $y_{1}=\theta \circ h_{n-2}^{\#}\left(\Lambda_{n-3}(g)\right)$ so again by (A), $y_{2}=\Lambda_{n-3}(g)$ is a solution of $\left(h_{n-3}, \cdots, h_{1} ; \Omega\right)(y)=0$. Continuing this way, we eventually obtain $y_{n-1}=g$ is a solution of $\Omega(y)=0$. 
10. Lemma. Let $c$ and $t$ be constants, $c \neq 0$ and $t>0$. Let $W$ be any function $\sim c x^{-1+t}$ in some $F(J)$, and let $f(\phi)=\cos (t \phi+\arg c)$ for $-\pi<\phi<\pi .(f(\phi)$ is called the indicial function for $W$, and was introduced in [6, \$61].) Then:

(a) If $J_{1}$ is any subinterval of $J$ on which $f(\phi)<0$ (respectively, $f(\phi)$ $>0)$, then for all $\alpha, \exp \int W \prec x^{\alpha}\left(\right.$ respectively, $\left.\exp \int W \succ x^{\alpha}\right)$ in $F\left(J_{1}\right)$.

(b) If $J_{2}$ is any subinterval of $J$ on which $f(\phi)$ is never zero, then for any function $H$, which in $F\left(J_{2}\right)$ is $\sim$ to a complex logarithmic monomial, the equation $\theta y=H \exp \int W$ possesses a solution of the form $y=G \exp \int W$, where $G$ is $\sim$ to a complex logarithmic monomial in $F\left(J_{2}\right)$. (In fact $G \sim H /(x W)$.)

Proof. (a) $z_{0}=\exp \int W$ is a solution of $z-\left(z^{\prime} / W\right)=0$. Assume $f(\phi)$ $<0$ on $J_{1}$. In this case it is proved in [3, p. 271], that any solution of $z-\left(z^{\prime} / W\right)=0$ is $\prec 1$ in $F\left(J_{1}\right)$ so $z_{0} \prec 1$. We now show that $z_{0} \prec x^{\alpha}$ for all real $\alpha$. Assume the contrary. Then the set $A$ of all real $\alpha$ for which $z_{0} \prec x^{\alpha}$, is nonempty (since it contains $\alpha=0$ ) and is bounded below. Letting $\beta$ be the greatest lower bound of $A$, we clearly have $z_{0}$ $\prec x^{\beta+\epsilon}$ for all $\epsilon>0$. Thus $z_{0}^{\prime} \prec x^{\beta+\epsilon-1}(\S 2(b))$. But $z_{0}=z_{0}^{\prime} / W$ and $W \sim c x^{-1+t}$. Thus $z_{0} \prec x^{\beta+\epsilon-t}$ for all $\epsilon>0$. Taking $\epsilon=t / 2$, we obtain $z_{0} \prec x^{\beta-t / 2}$ which contradicts the definition of $\beta$. Hence $z_{0} \prec x^{\alpha}$ for all $\alpha$ when $f(\phi)<0$.

If now $f(\phi)>0$, let $V=-W$. Then $V \sim-c x^{-1+t}$, so the indicial function for $V$ is $-f(\phi)$ which is strictly negative. Hence by the previous case, $\exp \int V \prec x^{\alpha}$ for all real $\alpha$, so $\exp \int W \succ x^{\alpha}$ for all $\alpha$.

(b) Let $H$ be given, $H \sim M$, where $M$ is a complex logarithmic monomial. Under the change of dependent variable, $y=u H \exp \int W$, the equation $\theta y=H \exp \int W$ is equivalent to,

$$
x H u^{\prime}+x\left(H W+H^{\prime}\right) u=H .
$$

Since $H \sim M, H^{\prime} / H$ is $\prec x^{-1}$ or $\approx x^{-1}$ and so is $\prec W$ (see $\S 2(\mathrm{~b})$ ). Thus $H W+H^{\prime} \sim H W$ so in some element of $F\left(J_{2}\right), H W+H^{\prime}$ is nowhere zero. Thus (1) is equivalent to,

$$
u-\left(u^{\prime} / V\right)=-(x V)^{-1}
$$

where $V=-\left(W+\left(H^{\prime} / H\right)\right)$. Hence $V \sim-W$. The indicial function for $V$ is $-f(\phi)$ and so is nowhere zero on $J_{2}$. It is proved in $[3$, p. 271] that for such a $V$, an equation of the form $u-\left(u^{\prime} / V\right)=E$ (where $E \prec 1)$ always possesses a solution $\prec 1$ in $F\left(J_{2}\right)$. Since $-(x V)^{-1} \prec 1$, there exists $u_{0} \prec 1$ in $F\left(J_{2}\right)$ satisfying equation (2). Thus,

$$
u_{0}=\left(u_{0}^{\prime} / V\right)-(x V)^{-1} \text {. }
$$


Since $u_{0} \prec 1, u_{0}^{\prime} \prec x^{-1}$ (see $\left.\S 2(\mathrm{~b})\right)$. Thus $\left(u_{0}^{\prime} / V\right) \prec(x V)^{-1}$ so by (3), $u_{0} \sim-(x V)^{-1}$ in $F\left(J_{2}\right)$. Hence $u_{0} H \sim-M /(x V)$ in $F\left(J_{2}\right)$. Since $y_{0}$ $=u_{0} H \exp \int W$ is a solution of $\theta y=H \exp \int W$, this proves (b).

11. Lemma. A ssume the hypothesis of $\$ 6$ and let $W$ be as in Lemma 8. Let $f(\phi)$ be the indicial function of $W$, and let $J$ be a subinterval of $I$ on which $f(\phi)$ is never zero. Then in $F(J)$, the equation $\Omega(y)=0$ possesses a solution of the form $y^{*}=H$ exp $\int W$, where $H$ is $\sim$ to a complex logarithmic monomial in $F(J)$. Furthermore, if on $J, f(\phi)<0$ (respectively, $f(\phi)>0)$, then for all real $\alpha, y^{*} \prec x^{\alpha}\left(\right.$ respectively, $\left.y^{*} \succ x^{\alpha}\right)$ in $F(J)$.

Proof. (In this proof, $L_{i}$ and $N_{i}$ will denote complex logarithmic monomials.) By Lemma 9, any function $y^{*}$ such that $\Lambda_{n-1}\left(y^{*}\right)$ $=\exp \int W$, will be a solution of $\Omega(y)=0$. We now construct such a $y^{*}$ by successive integrations using Lemma 10(b). By Lemma 10(b), there exists $G_{1} \sim L_{1}$ in $F(J)$ such that $z_{1}=G_{1} \exp \int W$ is a solution of $\theta z=\exp \int W$. Let $y_{1}=h_{n-1} z_{1}$. Thus, $\theta \circ h_{n-1}^{*}\left(y_{1}\right)=\exp \int W$, and $y_{1}$ $=H_{1} \exp \int W$ where $H_{1} \sim N_{1}$ by $\S 6(\mathrm{~A})$. Again by Lemma $10(\mathrm{~b})$, there exists $G_{2} \sim L_{2}$ such that $z_{2}=G_{2} \exp \int W$ is a solution of $\theta z=y_{1}$. Letting $y_{2}=h_{n-2} z_{2}$, we have $\theta \circ h_{n=1}^{\#} \circ \theta \circ h_{n-2}^{\#}\left(y_{2}\right)=\exp \int W$ and $y_{2}=H_{2} \exp \int W$ where by $\S 6(\mathrm{~A}), H_{2} \sim N_{2}$. Continuing this way, using Lemma 10(b), we obtain two sequences of functions $z_{2}, \cdots, z_{n-1}$ and $y_{2}, \cdots, y_{n-1}$ such that for each $j, z_{j}=G_{j} \exp \int W$ is a solution of $\theta z=y_{j-1}$, and $G_{j} \sim L_{j}$, and where $y_{j}=h_{n-j} z_{j}$. Thus clearly, $y_{j}=H_{j} \exp \int W$ where $H_{j} \sim N_{j}$ by $\S 6(\mathrm{~A})$. Let $y^{*}=y_{n-1}$ and $H=H_{n-1}$. Then clearly $y^{*}$ $=H \exp \int W$, and by construction $\Lambda_{n-1}\left(y^{*}\right)=\exp \int W$ so $\Omega\left(y^{*}\right)=0$ by Lemma 9. Let $N_{n-1}^{\sharp}=K x^{q}(\log x)^{r} \ldots$. Since $H \sim N_{n-1}$, it is easily verified that if $\alpha_{1}=1+\operatorname{Re}(q)$ and $\alpha_{2}=-1+\operatorname{Re}(q)$ then $H \prec x^{\alpha_{1}}$ while $H \succ x^{\alpha_{2}}$. Since $y^{*}=H \exp \int W$, we have $y^{*} \prec x^{\alpha_{1}} \exp \int W$ and $y^{*}$ $\succ x^{\alpha_{2}} \exp \int W$ in $F(J)$. If $f(\phi)<0$ then by Lemma 10(a), in $F(J)$, $\exp \int W \prec x^{\alpha}$ for all $\alpha$. Hence clearly $y^{*} \prec x^{\alpha}$ for all $\alpha$. Similarly, if $f(\phi)>0$, then by Lemma $10(\mathrm{a}), \exp \int W \succ x^{\alpha}$ for all $\alpha$, so clearly, $y^{*} \succ x^{\alpha}$ for all $\alpha$.

12. Conclusion of proof of theorem (\$4). If $W$ is as in Lemma 8 , and if $f(\phi)$ is the indicial function of $W$, then by Lemma $8(\mathrm{c}), f(\phi)$ has only finitely many zeros, $r_{1}<r_{2}<\cdots<r_{q}$ in $(a, b)$. Let $I_{1}$ be any of the intervals $\left(a, r_{1}\right),\left(r_{1}, r_{2}\right), \cdots,\left(r_{q}, b\right)$. Then by $\S 3$, e.f.d. in $F\left(I_{1}\right)$, there exists a complete logarithmic set of solutions of $\Omega(y)=0$. Letting $I$ be any subinterval of $I_{1}$, such that a complete logarithmic set of solutions $\left\{g_{1}, \cdots, g_{n-1}\right\}$ exists on $F(I)$, then in $F(I)$, by Lemma 11 , there is a solution $g^{*}$ of $\Omega(y)=0$, which is of the form 
$\mathrm{g}^{*}=H \exp \int W$ where $H$ is $\sim$ to a complex logarithmic monomial in $F(I)$. If $f(\phi)>0$ on $I, g^{*} \succ x^{\alpha}$ for all $\alpha$, while if $f(\phi)<0$ on $I, g^{*} \prec x^{\alpha}$ for all $\alpha$. Clearly the sets $\left\{g_{1}, \cdots, g_{n-1}, g^{*}\right\}$ exist e.f.d. in $F\left(I_{1}\right)$ (and e.f.d. in $F(a, b)$ ).

To conclude the proof of $\$ 4$, we must show that $\left\{g_{1}, \cdots, g_{n-1}, g^{*}\right\}$ is a linearly independent set.

Suppose for some constants $C_{1}, \cdots, C_{n}$ we have $\sum_{i=1}^{n-1} C_{i} g_{i}+C_{n} g^{*}$ $=0$. If $g^{*} \prec x^{\alpha}$ for all $\alpha$, then clearly $-C_{n} g^{*}$ is also trivial. Thus since $\sum_{i=1}^{n-1} C_{i} g_{i}=-C_{n} g^{*}$, we have $C_{1}=\cdots=C_{n-1}=0$ (see end of $\S 3$ ). Hence $C_{n} g^{*}=0$. Since $g^{*}=H \exp \int W, g^{*}$ is nonzero, so $C_{n}=0$ also. If $g^{*} \succ x^{\alpha}$ for all $\alpha$, then writing the dependence relation as $C_{n}=$ $-\sum_{i=1}^{n-1} C_{i}\left(g_{i} / g^{*}\right)$, we see that the right side of this relation $\rightarrow 0$, so $C_{n}=0$. Since $\left\{g_{1}, \cdots, g_{n-1}\right\}$ is linearly independent, $C_{1}=\cdots=C_{n-1}$ $=0$ also. Hence in both cases $\left\{g_{1}, \cdots, g_{n-1}, g^{*}\right\}$ is a fundamental set.

\section{BIBLIOGRAPHY}

1. S. Bank, On the instability theory of differential polynomials, Ann. Mat. Pura Appl. (to appear).

2. - An asymptotic analog of the Fuchs regularity theorem, J. Math. Anal. Appl. 16 (1966).

3. E. W. Chamberlain, Families of principal solutions of ordinary differential equations, Trans. Amer. Math. Soc. 107 (1963), 261-272.

4. E. Ince, Ordinary differential equations, Dover, New York, 1926.

5. W. Strodt, Contributions to the asymptotic theory of ordinary differential equations in the complex domain, Mem. Amer. Math. Soc., No. 13 (1954), 81 pp.

6. - Principal solutions of ordinary differential equations in the complex domain, Mem. Amer. Math. Soc., No. 26 (1957), 107 pp.

7. - Report on investigation in differential equations, Contract NSFG 12984 between the National Science Foundation and Columbia University, November, 1961.

UNIVERSITY OF ILLINOIS 